\section{FRI0681 CLINICAL VALIDATION STUDIES OF THE 2012 CLASSIFICATION CRITERIA FOR EARLY RHEUMATOID ARTHRITIS (ERA) IN A DOMESTIC MULTI-CENTER COHORT}

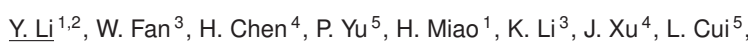
G. Zhang ${ }^{1}$, K. Xu ${ }^{1}$, L. Zhang ${ }^{1}$, R. Li ${ }^{2} .{ }^{1}$ Shanxi Academy of Medical Sciences Shanxi Dayi Hospital, Taiyuan; ${ }^{2}$ Rheumatology and Immunology, Peking University People's Hospital, Beijing; ${ }^{3}$ Xinxiang Central Hospital, Xinxiang; ${ }^{4}$ the Third Hospital of Hebei Medical University, Shijiazhuang; ${ }^{5}$ Kailuan General Hospital, Tangshan, China

Background: Recently, a new classification criteria for early rheumatoid arthritis (ERA) have been developed.

Objectives: To evaluate the value of 2012 classification criteria for early rheumatoid arthritis (ERA), 2010 ACR/EULAR classification criteria, and 1987 ACR classification criteria in the diagnosis of early RA.

Methods: Early arthritis patients with age more than 16 years, disease duration no more than 1 year, Cat least one joint swelling and tenderness were enrolled in a multicenter, open, cross-sectional study cohort. The patients were diagnosed as RA or other none RA disease by 2 trained experienced rheumatologists. Detailed recorded the clinical and laboratory parameters include disease duration, morning stiffness duration, RF, anti-CCP, ESR, CRP etc. The sensitivity and specificity of three RA classification criteria were compared by McNemar test, The areas under the ROC curve (AUC) of each RA classification criteria were analyzed using MedCalc software.

Results: A total of 310 patients were randomly enrolled in this study, including 182 ERA and 128 non-RA. The sensitivity (88.5\%) of ERA criteria were much higher than that of 1987 ACR criteria $\left(45.6 \%, \chi^{2}=75.013, P<0.0125\right)$, and not significantly different with the 2010 ACR/EULAR criteria $\left(91.8 \%, \chi^{2}=1.042\right.$, $P>0.05)$. The specificity of ERA criteria (91.4\%) were similar to those of 2010 ACR/EULAR criteria $\left(87.5 \%, x^{2}=1.8, P>0.05\right)$ and 1987 ACR criteria $(96.1 \%$, $\left.\chi^{2}=3.1, P>0.05\right)$. The AUC of ERA criteria was $0.962(95 \% \mathrm{Cl}: 0.934,0.980)$, which was slightly better than that of the 2010 ACR/EULAR criteria $[0.959(95 \%$ Cl: $0.931,0.978), Z=0.380, P=0.7038]$, and much higher than that of the 1987 ACR criteria [0.885 (95\% Cl: $0.845,0.919), Z=4.517, P<0.0001]$.

\begin{tabular}{|c|c|c|c|c|c|c|c|c|c|c|}
\hline \multirow{3}{*}{ Groups } & \multirow{3}{*}{ Cases } & \multirow{3}{*}{$\begin{array}{l}\text { Age(year) } \\
\bar{y} \pm z\end{array}$} & \multirow{2}{*}{\multicolumn{2}{|c|}{ Gender }} & \multicolumn{4}{|c|}{ Disease } & \multirow{2}{*}{\multicolumn{2}{|c|}{$\begin{array}{l}\text { Mouning } \\
\text { stiffness } \\
30 \mathrm{~mm}\end{array}$}} \\
\hline & & & & & \multirow{2}{*}{\multicolumn{2}{|c|}{$\begin{array}{c}\text { duration } \\
\text { (months) } \\
\bar{X} \pm z\end{array}$}} & \multirow{2}{*}{$\begin{array}{l}\text { SIC } \\
\bar{x} \pm s\end{array}$} & \multirow{2}{*}{$\begin{array}{l}\text { TJC } \\
\bar{y} \pm s\end{array}$} & & \\
\hline & & & Fem2le & $\%$ & & & & & & cases \\
\hline ERA & 182 & $48.2 \pm 14.0$ & 140 & 76.9 & \multicolumn{2}{|c|}{$6.7 \pm 3.9$} & $8.6 \pm 7.4$ & $11.4 \pm 7.7$ & & 74.7 \\
\hline Non-RA & 128 & $42.8 \pm 16.2$ & 49 & 38.3 & \multicolumn{2}{|c|}{$4.3 \pm 4.1$} & $2.0 \pm 4.1$ & $3.1 \pm 5.5$ & & 13.3 \\
\hline$P$ value & & 0.002 & \multicolumn{2}{|c|}{0.000} & \multicolumn{2}{|c|}{0.000} & 0.000 & 0.000 & \multicolumn{2}{|r|}{0.000} \\
\hline \multirow{2}{*}{ Groups } & \multirow{2}{*}{ Cases } & $\mathrm{ESR}(\mathrm{mm} / \mathrm{h})$ & \multirow{2}{*}{\multicolumn{2}{|c|}{$\begin{array}{c}\text { CRP(mgL) } \\
\bar{x} \pm s\end{array}$}} & \multicolumn{2}{|c|}{ IgM-RF $(+)$} & \multicolumn{2}{|c|}{ 放 $\mathrm{CCP}(+)$} & \multicolumn{2}{|r|}{ HAQ } \\
\hline & & $\bar{x} \pm s$ & & & 刹 & $\%$ & 列 & $\%$ & $\bar{M}$ & Q1-Q3 \\
\hline ERA & 182 & $39.7 \pm 25.5$ & \multicolumn{2}{|c|}{$22.0 \pm 28.2$} & 138 & 75.8 & 131 & 72.0 & 0.7 & $0.3 \cdot 1.2$ \\
\hline Non RA & 128 & $32.3 \pm 23.8$ & \multicolumn{2}{|c|}{$28.5 \pm 39.7$} & & 6.3 & 2 & 1.6 & 0.4 & 0.2 .0 .8 \\
\hline$P$ value & & 0.011 & \multicolumn{2}{|c|}{0.099} & \multicolumn{2}{|c|}{0.000} & \multicolumn{2}{|c|}{0.000} & \multicolumn{2}{|r|}{0.001} \\
\hline
\end{tabular}

SJC: Swollen joimt counts; TJC: Tender joint courst $t$

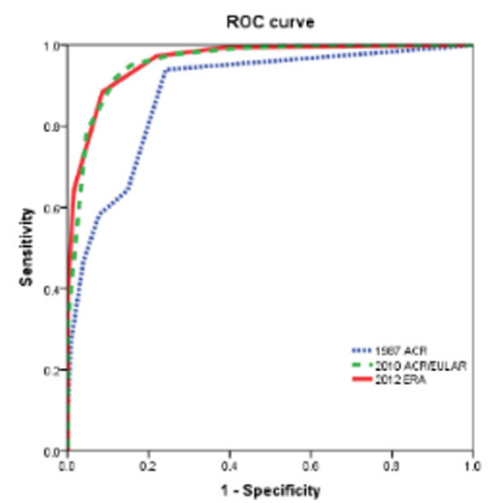

Fig.1 Comparison of three classification criteria of rheumatoid arthritis

Conclusions: Overall evaluation, the diagnostic value of ERA criteria is better than 1987 ACR and 2010 ACR/EULAR criteria in early rheumatoid arthritis. Compared to 2010 ACR/EULAR classification criteria, ERA criteria is obviously more simple and practical.

References:

[1] Li R, Sun J, Ren LM, et al. Epidemiology of eight common rheumatic diseases in China: a large-scale cross-sectional survey in Beijing[J]. Rheumatology, 2012, 51(4): 721-729.

[2] Radner H, Neogi T, Smolen JS, et a1. Performance of the 2010 ACR/EULAR classification criteria for rheumatoid arthritis: a systematic literature review[J]. Ann Rheum Dis, 2014, 73(1): 114-123.
[3] Cader MZ, Filer A, Hazlehurst J, et al. Performance of the 2010 ACR/EULAR criteria for rheumatoid arthritis: comparison with 1987 ACR criteria in a very early synovitis cohort[J]. Ann Rheum Dis, 2011, 70(6): 949-955.

[4] Ye H, Su Y, Li R, et al. Comparison of three classification criteria of rheumatoid arthritis in an inception early arthritis cohort[J]. Clinical Rheumatology, 2016, 35(10): 2397-401.

[5] Zhao JX, Su Y, Liu XY. et al. Classification criteria of early rheumatoid arthritis and validation of its performance in a multi-centre cohort[J]. Clinical and Experimental Rheumatology, 2014, 32(5): 667-673.

Disclosure of Interest: None declared

DOI: 10.1136/annrheumdis-2017-eular.3112

\section{FRI0682 FOLLOW-UP OF TREATMENT RESPONSE WITH DYNAMIC DOPPLER ULTRASOUND IN RAYNAUD'SPHENOMENON}

U. Toprak ${ }^{1}$, Z. Ozbalkan ${ }^{2}$, M. Edugan ${ }^{3}$, S. Parlak ${ }^{4}$, S.C. Sandıkci ${ }^{2}$, T. Kaya ${ }^{4}$, S. Saylisoy ${ }^{5} .{ }^{1}$ Department of Radiology, Suleyman demirel University, Isparta; ${ }^{2}$ Department of Rheumatology, Ankara Numune Education and Research Hospital, Ankara; ${ }^{3}$ Department of Rheumatology, Istanbul University, Capa School of Medicine, Istanbul; ${ }^{4}$ Department of Radiology, Ankara Numune Education and Research Hospital, Ankara; ${ }^{5}$ Department of Radiology, Eskisehir Osmangazi University, School of Medicine, Eskisehir, Turkey

Background: This study aims to investigate the role of flow parameters obtained by dynamic Doppler ultrasound in the objective follow-up of treatment response in Raynaud's phenomenon (RP) cases.

Methods: The study included newly diagnosed 33 patients with primary RP (PRP), 31 patients with secondary RP (SRP), and 26 healthy controls. The control group was evaluated with Doppler once, while the patients before treatment and on the third month of thet reatment. Baseline and post-cold provocation diameter (BD, $\mathrm{CPD}, \mathrm{mm}$ ) andflowvolume (BFV, CPFV, $\mathrm{mL} / \mathrm{min}$ ); post-coldprovocationflowstarting time (FST, min), and flowv olume normalizing time (FVNT, min) were recorded.

Statistical analysis: for disturubution of thes ex of the groups: chi-square test, for analysing age distrubution among the groups One-way ANNOVA; for analysing the pre and post treatment doppler results: Wilcoxon test and fo rcomparison of the PRP and SRP post treatment values to control group; Krusal Wallis test were used. A p value less than 0,05 was considered statistically significant.

Results: Before-after treatment, there was no significant improvement in the BD in both PRP and SRP groups $(0.79 \pm 0.17-0.82 \pm 0.19$ vs. $0.66 \pm 0.13-0.68 \pm 0.14$ PRP vs. SRP, respectively), while FST did not significantly improve in the PRP group (1.15 $\pm 2.27-0.61 \pm 1.41$ vs $3.13 \pm 4.81-1.58 \pm 2.36)$ ( $p>0.05)$. A significant improvement was observed in baseline flow volüme $(3.08 \pm 2,96$ vs $3.91 \pm 3.39$ (p: 0,002), flow volume normalization time $(7.24 \pm 7.60$ vs $3.84 \pm 3.39)$ ( $p: 0,0001)$, after cold provocation flow volume $(1.18+1,26$ vs $2.17+2.16)$ (p: 0.0001$)$, after cold provocated diameter $(0.63 \pm 0.15$ vs $0.70 \pm 0.16)(p: 0,005)$ in PRP group after treatment.

In SRP group, only baselined iameter changes were not influenced by the treatment, all other post treatmen tparameters were impoved in all SRP cases including baseline flow volume $(2.14 \pm 1.94$ vs $2.80 \pm 2.15)$ ( $p: 0,009)$, after cold provocation diameter $(0.56 \pm 0.15$ vs $0.63 \pm 0.13)$ (p: 0.004$)$, after cold provocation flow volume $(1.07 \pm 1.40$ vs $1.46 \pm 1.67)$ ( $p$ : 0,004$)$, flow starting time $(3.13 \pm 4.81$ vs $1.58 \pm 2.36)$ (p: 0,021$)$ and flow volume normalisation time $(9.58 \pm 8.49$ vs 4.32 \pm 3.56$)$ (p: 0,0001).

There was an improvement in parameters after the treatment in both RP groups comparing by the control groups $(\mathrm{p}<0.01)$.

Conclusions: Doppler ultrasound is an objective, cost-effective, safe (does not include radiation), and easy-to-use method in thefollow-up of RP patients on macrovascular level with or without cold provocation before and after treatment. Acknowledgements:

Keywords: Raynoud Phenomenon, treatment, Doppler ultrasound

Disclosure of Interest: None declared

DOI: 10.1136/annrheumdis-2017-eular.3122

FRIDAY, 16 JUNE 2017

Epidemiology, risk factors for disease or disease progression

\section{FRI0683 CAUSES OF DEATH IN 350 PATIENTS WITH SYSTEMIC AUTOIMMUNE RHEUMATIC DISEASES (SARD)}

J.G. Ovalles-Bonilla ${ }^{1,2}$, O. Fernández $^{3}$, J. Martínez-Barrio ${ }^{1}$, L. Valor ${ }^{1,2}$ D. Hernández ${ }^{1,2}$, I. Janta ${ }^{1}$, B. Serrano ${ }^{1}$, C. Sáenz ${ }^{1}$, R. González ${ }^{1}$, M. Correyero ${ }^{1}$, L. García ${ }^{1}$, A. López ${ }^{1}$, A. Silva ${ }^{1}$, J.C. Nieto ${ }^{1}$, C. González ${ }^{1,4}$ I. Monteagudo ${ }^{1}$, F.J. López-Longo ${ }^{1,4}$. ${ }^{1}$ Rheumatology, Hospital General Universitario Gregorio Marañón; ${ }^{2}$ Instituto de Investigación Sanitaria Gregorio Marañón, Madrid; ${ }^{3}$ Rheumatology, Hospital Universitario de Basurto, Bilbao;

${ }^{4}$ Facultad de Medicina, Universidad Complutense de Madrid, Madrid, Spain

Background: The major SARD have an increased mortality compared to the general population. It is well known that the main causes of death in Systemic Lupus Erythematosus (SLE) are infections (INF), cardiovascular events (CV), neoplasia 
(NEO) and disease activity. However, the compared mortality of Mixed Connective Tissue Disease (MCTD), Systemic Sclerosis (SSc), Poly/Dermatomyositis (PM/DM), overlap syndromes (OS), Sjögren's syndrome (SS), Antiphospholipid syndrome (APS), systemic vasculitis (SV), and undifferentiated or incomplete Connective Tissue Disease (UCTD) is poorly described.

Objectives: To analyze the causes of death and the autoantibodies (AAB) profile among the SARD.

Methods: This was a single center, prospective and observational study. Mortality by all causes and relationship with $A A B$ profile were analyzed in patients diagnosed of SLE, MCTD, SSc, PM/DM, OS (simultaneous or sequential criteria of 2 or more SARD), SS, APS, SV and UCTD or incomplete SARD (at least one clinical criterion of the classification criteria and a related antibody of any of the SARD). Data were obtained from the "Systemic Autoimmune Rheumatic Diseases Registry" of a tertiary referral hospital from 1986 to 2016. Patients with rheumatoid arthritis were excluded. The SARD registry counts with the institutional review board approval.

Results: 1750 patients were included, of whom $1453(83 \%)$ were women. Five hundred fifty six SLE, 125 SSc, 111 PM/DM, 91 OS, 90 MCTD, 250 SS, 71 APS, 211 SV, 117 UCTD and 128 losses to follow-up, the global follow up rate was $92.7 \%$. A global mortality of $350(20 \%)$ cases was observed: 101 INF $(28,8 \%), 89$ CV $(25,4 \%), 51 \mathrm{NEO}(14,5 \%), 45$ due to disease activity (12,8\%), 41 other causes $(11,7 \%)$ and 23 from unknown causes $(6,5 \%)$. Table 1 shows detailed mortality causes compared by diseases. A higher mortality was associated $(p<0,05)$ with older patients (71 years, 20-96), SV (OR 3,65), male patients (OR 1,95), SSC/PM/DM (OR 1,76), MCTD (OR 1,6) and OS (OR 1,43). AAB to pANCA (OR 4,43 ), anti-topoisomerase I (OR 3,64), myositis-specific AAB (OR 3.0), cANCA (OR 2,19) and anticardiolipin (OR 1,89) were associated with poorer survival. A higher survival rate was observed in patients with SLE (OR 1,7), SS (OR 1,69) and UCTD (OR 15,57) $(\mathrm{p}<0,05)$.

\begin{tabular}{lcccccccc}
\hline CAUSES & SLE, \% & SSC, $\%$ & MCTD, \% & SV, \% & PM/DM, \% & OS, \% & SS, \% & APS, \% \\
\hline CV & $\mathbf{3 0 , 8 6}$ & $\mathbf{2 7 , 0 2}$ & $\mathbf{4 2 , 3}$ & 25,55 & 18,18 & 16,12 & 17,64 & 21,4 \\
INF & 27,16 & 21,62 & 3,84 & $\mathbf{3 6 , 6 6}$ & $\mathbf{3 3 , 3 3}$ & $\mathbf{4 5 , 1 6}$ & 23,52 & 21,4 \\
NEO & 14,81 & 13,51 & 15,38 & 8,88 & 18,18 & 9,67 & $\mathbf{2 6 , 4 7}$ & 14,28 \\
ACTIVITY & 9,87 & 18,91 & 19,23 & 11,11 & 12,12 & 19,35 & 11,76 & $\mathbf{2 8 , 5 7}$ \\
OTHER & 8,64 & 13,51 & 11,53 & 15,55 & 9,09 & 3,22 & 8,82 & 0.00 \\
UNKNOWN & 8,64 & 5,40 & 7,69 & 2,22 & 9,09 & 6,45 & 11,76 & 14,28 \\
\hline
\end{tabular}

Conclusions: The main causes of death among SARD patients are CV (MCTD, SLE, and SSC), severe infections (OS, SV, and PM/DM), disease activity (APS) and neoplasia (SS). A higher mortality is observed among ANCA positive SV, antitopoisomerase I positive SSC, MCTD, OS, anticardiolipin and myositis-specific positive patients.

Disclosure of Interest: None declared

DOI: 10.1136/annrheumdis-2017-eular.5564

\section{FRI0684 EARLY TREATMENT WITH METHOTREXATE IMPROVES ALL-CAUSE AND CARDIOVASCULAR SURVIVAL AMONG AN INCEPTION COHORT OF SENIORS WITH RHEUMATOID ARTHRITIS}

J. Widdifield ${ }^{1}$, J.M. Paterson ${ }^{2}$, A. Huang ${ }^{2}$, S. Bernatsky ${ }^{1}$ on behalf of CANAIM ${ }^{1}$ Mcgill University, Montreal; ${ }^{2}$ Institute for Clinical Evaluative Sciences, Toronto, Canada

Background: We previously observed that incident RA patients have an increased risk of all-cause and cardiovascular disease (CVD) mortality relative to the general population in Ontario. ${ }^{1}$

Objectives: To evaluate the effect of treatment and other factors on all-cause and CVD mortality among incident senior RA patients.

Methods: We studied incident RA patients within the population-based Ontario Rheumatoid Arthritis Database (ORAD) who were diagnosed after their 65th birthdate (ensuring comprehensive drug coverage) between 2000 and 2013. Patients are included in ORAD if they received 3 physician billing RA diagnosis codes (with at least one provided by a MSK specialist) over 2 yrs OR at least 1 hospital RA diagnosis code. All patients were followed from cohort entry (when all case definition criteria were met) until death, out-migration, or end of study period (Dec 2013). Two multivariable Cox regression models were performed to estimate hazard ratios (HRs) for each outcome (all-cause and CVD mortality, separately), exploring the effects of both baseline/early treatment (within the year preceding index date) and time-varying medication exposures over the entire duration of follow-up (for methotrexate, other DMARDs, anti-TNFs, COXIBS, NSAIDs, glucocorticosteroids, statins, antihypertensives), baseline comorbidities (within 3 yrs prior to index date), time-varying development of extra-articular manifestations (as proxy for disease severity), healthcare use, and demographics (age, sex, rurality, SES).

Results: 28,172 incident RA patients were followed for 141,072 person years. During follow-up, $8,848(31 \%)$ patients died with $1,419(5 \%)$ deaths due to CVD, corresponding to an all-cause rate of 62.7 deaths $(95 \% \mathrm{Cl} 61.4,64.0)$, and 10.1 deaths due to CVD $(95 \% \mathrm{Cl} 9.5,10.6)$ per 1,000 patient-years, respectively. In our multivariable analysis focused on all-cause mortality, early treatment with methotrexate [HR $0.90(95 \% \mathrm{Cl}$ 0.85,0.96)] and other DMARDs [HR $0.92(95 \%$ $\mathrm{Cl} 0.87,0.97)]$ were associated with a lower mortality risk. For CVD mortality, early treatment with methotrexate was associated with lower risk estimates [HR $0.71(95 \% \mathrm{Cl} 0.60,0.83)$ ], which was not clearly seen with other DMARDs [HR $0.94(95 \% \mathrm{Cl} 0.82,1.08)]$. Use of COX-II inhibitors and NSAIDs at baseline were associated with lower HR for all-cause and CVD mortality risk, though we were unable to detect clear associations with greater use during follow-up. Greater cumulative exposure to glucocorticosteroids, comorbidities, and extra-articular manifestations of RA were associated with increasing risk of all-cause and CVD mortality.

Conclusions: Patients with early exposure to methotrexate had a lower risk of all-cause and CVD mortality. Our findings support the hypothesis that early treatment, by reducing inflammation, may help improve survival in RA. However, residual confounding cannot be ruled out.

\section{References:}

[1] Widdifield J, et al. Risk of Vascular Mortality in Seniors with New-Onset Rheumatoid Arthritis. Arthritis Rheumatol 2016; 68 (suppl 10).

Disclosure of Interest: None declared

DOI: 10.1136/annrheumdis-2017-eular.3034

\section{FRI0685 THE PRESENCE OF RHEUMATOID FACTOR IS ASSOCIATED WITH LOWER BONE MASS IN KOREAN HEALTH SCREENING MALE SUBJECTS WITHOUT CLINICALLY APPARENT ARTHRITIS}

J. Hwang ${ }^{1}$, J.K. Ahn ${ }^{2}$, Y.H. Eun ${ }^{3}$, E.-J. Park ${ }^{4}$, J. Lee ${ }^{3}$, E.-M. Koh ${ }^{3}$, H.-S. Cha ${ }^{3}$ ${ }^{1}$ Department of Internal Medicine, National Police Hospital; ${ }^{2}$ Department of Medicine, Kangbuk Samsung Hospital, Sungkyunkwan University School of Medicine; ${ }^{3}$ Department of Medicine, Samsung Medical Center, Sungkyunkwan University School of Medicine, Seoul; ${ }^{4}$ Department of Medicine, Jeju National University Hospital, Jeju, Korea, Republic Of

Background: Close relationship between the immune and skeletal systems has been recognized through the bone loss in rheumatoid arthritis (RA). Rheumatoid factor (RF) is present in approximately $70-80 \%$ of RA patients, which is an autoantibody directed against the $\mathrm{Fc}$ component of $\mathrm{IgG}$ and associated with osteoporosis and reduced bone mineral density (BMD) in RA. RF is also found nonspecifically in chronic inflammatory condition such as sarcoidosis, hepatitis $\mathrm{B}$ or $\mathrm{C}$, and tuberculosis. However, the influence of RF to bone loss is scarcely known in subjects without any specific medical problem.

Objectives: This cross-sectional study aimed to investigate the association between the presence of RF and BMD in Korean healthy male subjects without any history of joint disease.

Methods: Of the 84,344 males who had undergone a comprehensive health checkup program in 2012, 1,390 healthy subjects were recruited, whose BMD and RF results were available. Subjects with history of diabetes, kidney disease, thyroid disease, and malignancy, and taking medicine regarding these diseases, osteoporosis, and arthritis were excluded based on self-reported questionnaire. The RF titer $\geq 20 \mathrm{IU} / \mathrm{ml}$ was considered positive. BMD was categorized into 3 groups based on T-score; normal (T-score $\geq-1.0)$, osteopenia $(-1.0>$ T-score $>$ -2.5 ) and osteoporosis (T-score $\leq-2.5$ ). The association between the presence of $\mathrm{RF}$ and $\mathrm{BMD}$ was assessed by multiple linear regression analysis.

Results: Of 1,390 males, the mean age was $52.8 \pm 10.9$ years (range, $22-83$ ) and RF was positive in 64 subjects $(4.6 \%)$. Demographics including smoking history, alcohol consumption, the frequency of vigorous exercise and body mass index (BMI), and laboratory data were not different between RF-positive and -negative subjects except hepatitis B surface antigen, which was more frequently seen in RF-positive subjects $(15.6 \%$ vs. $4.3 \%, p=0.001)$. Low bone mass (osteopenia and osteoporosis) of lumbar spine was more prevalent in subjects aged 50 or more compared with those younger than 50 years $(28.0 \%$ vs. $10.7 \%$, $p<0.001$ ) while no differences of femur neck and total hip. RF-positive subjects had significantly lower BMD compared to RF-negative subjects in lumbar spine $\left(1.10 \pm 0.18 \mathrm{~g} / \mathrm{cm}^{2}\right.$ vs. $\left.1.17 \pm 0.16 \mathrm{~g} / \mathrm{cm}^{2}, p=0.002\right)$ but neither in femur neck nor total hip. In subjects with higher titer RF ( $\geq 40 \mathrm{IU} / \mathrm{ml})$, the mean BMD of lumbar spine was significantly decreased than those with lower titer RF (one-way ANOVA, $F(3,1190)=3.527, p=0.015)$. After adjusting for multiple confounders such as age, BMI, glomerular filtration rate, serum concentration of calcium, phosphorus, and uric acid, and lifestyle factors (drinking, smoking, and physical exercise), RF positivity was negatively associated with BMD at lumbar spine $(B=-0.055$ and $S E=0.027, p=0.039$ ).

Conclusions: Our results provide epidemiological evidence that the presence of RF could have an unfavorable impact on bone density in apparently healthy male subjects. Additional studies to elucidate the osteoimmunological mechanism of $\mathrm{RF}$ are warranted.

Disclosure of Interest: None declared

DOI: 10.1136/annrheumdis-2017-eular.2645 